\title{
AIM: AN INTERACTIVE ASHTRAY TO SUPPORT BEHAVIOR CHANGE THROUGH GAMIFICATION
}

\author{
Huang, Siyuan; Scurati, Giulia Wally; Elzeney, Mostafa; Li, Yujie; Lin, Xiaofeng; Ferrise, \\ Francesco; Bordegoni, Monica \\ Politecnico di Milano, Italy
}

\begin{abstract}
Littering is a highly diffused anti-environmental and anti-social behavior, especially among young people. Furthermore, cigarette butts are one of the most littered items and are responsible for both severe environmental damages and high clean up expenses. The aim of this project is to design an interactive ashtray for the campus environment to limit the cigarette butts littering behavior in an engaging and effective way. Qualitative and quantitative data are collected. Coded observations were implemented through the research process, including the 2 pre (without the prototype) and 2 pros (with the prototype) sessions. Also, user experience test and one to one interview were conducted for deepening the understanding of the littering phenomenon and the reasons behind in the behavior among young people. The prototype indeed reduced the number of cigarette butts littering among observed behaviors of 156 students, especially in male sample. Final results indicate the behavior change of disposers is moderated by other factors, as the environmental cleanliness. Future development is also discussed.
\end{abstract}

Keywords: Sustainability, User centred design, Multisensory product experience, Design for Behavior Change, Gamification

\section{Contact:}

Scurati, Giulia Wally

Politecnico di Milano

Mechanical Engineering

Italy

giuliawally.scurati@polimi.it

Cite this article: Huang, S., Scurati, G.W., Elzeney, M., Li, Y., Lin, X., Ferrise, F., Bordegoni, M. (2019) 'AIM: An Interactive Ashtray to Support Behavior Change through Gamification', in Proceedings of the 22nd International Conference on Engineering Design (ICED19), Delft, The Netherlands, 5-8 August 2019. DOI:10.1017/dsi.2019.388 


\section{INTRODUCTION}

Design for Sustainable Behavior is a growing field which uses design as a mean to modify those human behaviors which are damaging the environment by causing waste of resources or pollution. Littering is one of those behaviors since it pollutes both the natural and civic environments, which is why it is considered not only anti-environmental but also anti-social. Cigarette littering, in particular, is acknowledged to be responsible for metal contamination and cigarette butts are hardly biodegradable. Therefore, it causes both environmental damage, affecting ecosystems and organism, and economic damage, as the cleanup is highly expensive (Rath et al., 2012). However, littering is still a common collective behavior, as it can be observed in urban open public spaces.

The design of trash bins and ashtrays can affect the littering behavior: some studies suggest that by improving the receptacle appearance it is possible to reduce littering (Geller et al., 1979; Finnie, 1973). According to Montazeri et al. (2015) using the green color can be an effective prompt to recycle: in their study, $88 \%$ of the participants involved used the recycle bin when it was green, while only the $52 \%$ used it when it was gray. Also, bins which convey messages related to both social and personal norms (related to the self-concept), can reduce littering (De Kort et al., 2008).

The aim of this work is to present the design of an ashtray for the campus outdoor environment, where cigarette littering is often a common behavior. The difficulty of changing the littering behavior, especially in this context, is due to the following facts:

- Cigarettes, small objects, and organic items are more likely to be littered (Wever et al., 2010). Also, cigarettes are sometimes not perceived as litters (Rath et al., 2012).

- $\quad$ Young people - as university students - litter more than middle-aged and elderly people (Schultz et al., 2013; Bator et al., 2011; Finnie, 1973; De Kort et al., 2008).

- Smoking is a strongly habitual behavior, which smokers perform several times per day, leading the related gestures to be highly automated. Some smokers reported having found themselves smoking without even remembering the decision to do that (Jager, 2003).

- The littering behavior of a smoker is often influenced negatively by the other smokers' behavior, as signs of past littering causes further littering (Schultz et al., 2013; Cialdini et al., 1990).

As claimed in the last point, social aspects play a fundamental role in littering behavior, especially in the case of cigarette littering. In fact, smoking is often considered as a social activity: it is associated with the ideas of having a break, enjoying a talk with friends or colleagues, as it often happens in campus and workplaces. In this work, a user-centered approach was adopted for achieving the goal of motivating smokers not to litter their cigarette butts in a playful way. The adopted strategy is to re-design products by adapting them to people, allowing them to perform actions in a natural way. Specifically, the new design faces this issue in a novel way: rather than requiring a radically different behavior, it aims at turning the negative action of throwing cigarettes on the ground into a positive one. To do this, the ashtray was designed to encourage people gathering around it, throwing butts in a spontaneous manner, including elements of gamification to involve a small group of smokers and leverage social influence. Gamification is defined by Deterting et al. (2011) as "an informal umbrella term for the use of game design elements in non-gaming systems to improve the user experience and engagement". The use of gamification techniques can be highly motivating and persuasive, supporting designers in shaping users' behavior: this represents a great advantage when the aim of a design is to support sustainable actions and choices (Deterting et al., 2011). However, the definition we cited also expresses the comprehensive and vague character of gamification, which involves a variety of approaches, techniques and research fields. In this scenario, orienting themselves and implementing gamification strategies is not a trivial task for designers. In Sections 2 and 3 we describe and then classify case studies regarding ashtrays and bins, mentioning design principles, interaction levels and social factors, furthermore we relate them to different contexts and users. Then, basing on our specific context and users, we selected and combined these factors, obtaining requirements for our design, which was prototyped and tested.

\section{TRASH BINS AND ASHTRAYS FOR BEHAVIOR CHANGE}

One of the main reasons people do not perform pro-social or pro-environmental behaviors is that they find it difficult in terms of the various type of resources (e.g. physical, mental, economical) it requires to be accomplished, as described by Fogg (2009). Then, an approach to behavior change is enabling the right behavior by making it more convenient than the wrong one. In the case of littering, convenience 
can be depending on the availability and distance of the litter receptacle from the user, which is a determining factor (Schultz et al., 2013; Bator et al., 2011; Finnie, 1973). A possible solution is then the one of making the trash bin easier to find, for instance through green footprints leading to trash bins. However, it is interesting to notice that a similar trigger, using arrows leading to stairs, did not have the same success in making people use the stairs instead of the escalator: this might be caused by the presence of a social norm to be violated in the first case but not in the second (Hansen, 2012). This fact recalls the importance of social factors: as already mentioned, littering is most likely to happen when littered objects are present in the environment since people are influenced by others' behavior. However, this dynamic could also be reversed, taking advantage of social influence. For instance, a transparent trash bin makes the previous and proper behavior evident as a social norm, furthermore, it makes it easier to collect trash correctly (Matsumura et al., 2015). Moreover, according to Fogg (2009) social factors also affect a behavior simplicity. In fact, he defines social deviance as having a behavior which does not conform to the rules of society or to a specific situation. In the case of the transparent bin, the negative behavior is made more difficult by highlighting the users' social deviance, then the correct behavior appears easier. This concept has also been used in private contexts: the BinCam system makes the users' food waste and recycling behavior visible and public through social networks (Thieme et al., 2012). Conversely, the Trash Game uses an application to share pictures of litters to educate crowds regarding correct disposal, without revealing the user's identity. In fact, the system questions the players regarding the correctness of the chosen bin (Lessel et al., 2015).

Drop Pit (https://www.thedroppit.eu/), is another case which reduces littering by making the behavior easier: users need to perform the simple and habitual action of throwing cigarettes on the ground over street grates. The ashtray resembles indeed a street grate which has been intentionally designed to collect cigarettes butts. A different way to guide a behavior is using incentive mechanism, for example, simple game elements like emoticons and sounds as described by Berengueres et al. (2013). Emoticons can also raise users' empathy towards the bin as for the anthropomorphic bin (Tan et al., 2016), which has human-like features and reactions to users' behavior. FUMO (https://www.fastcompany.com/3030479/meet-fumo-the-silliest-possible-way-to-fixcigarette-littering) is instead an interactive ashtray which plays different music and light patterns each time a smoker throws a cigarette butt inside it.

Again, this last case also shows how in public and crowded spaces the social aspects play an important role. However, this time it is not due to a fear of disapproval, but rather to raising involvement and participation. In fact, people are attracted by the trash bin when they notice the others' experiences, as it can also be observed in the successful examples provided by the Fun Theory (Kim, 2015). For instance, the World's Deepest Bin produces a sound when someone throws garbage into it, while the Bottle Bank Arcade invites to play an arcade game with bottles (Kim, 2015). Gamification can also involve multiple players at the same time, as for the TetraBin, which displays a collaborative Tetris game reacting to the litters disposed of (http://www.tetrabin.com/). Finally, other concepts make people compete as part of social groups, leveraging people identity. The Ballot Bin (https://ballotbin.co.uk/) displays a question and requires people to vote by inserting the cigarette butt in the slot corresponding to their answer. An example is the one of challenging two football teams' supporters.

\section{TRASH BIN AND ASHTRAYS: A CLASSIFICATION}

As described, there are various approaches to address the littering behavior, which can be classified depending on the way the behavior is triggered. Among them, social factors appear to be common because of the social character of the littering behavior, and especially of the smoking actions. Social factors can be distinguished into two main categories:

- Pressure: users feel that their actions will be noticed and judged by others;

- Involvement: users are stimulated to be active participants in performing the correct behavior and eventually interact with other users;

However, the case studies can also be classified according to the levels of complexity of the interaction depending on the context, situation and target users. The interaction complexity is defined as follow:

- Low: the bin or ashtray is simply designed to attract the users, requires them to perform the usual basic actions to interact with it and does not provide any immediate feedback. 
- Medium: the bin or ashtray requires the user to perform the usual basic actions to interact with it but then provides immediate feedback which raise the users' curiosity and surprise.

- High: the bin or ashtray provides inputs or requires the user to perform different actions to interact with it, then also provides immediate feedback which may vary depending on the user action.

Table 1 compares twelve case studies that have been discussed in the previous Section, considering the social factors they address and evaluating their interaction complexity. Another important aspect to make this comparison is the one of the context, which can be public (indoor/outdoor-urban) or private.

Table 1. Classification of existing trash bins and ashtrays

\begin{tabular}{|c|c|c|c|c|c|c|c|}
\hline Name & Year & $\begin{array}{c}\text { Target } \\
\text { Behavior }\end{array}$ & Context & Social factor & Short description & Principle & $\begin{array}{l}\text { Interaction } \\
\text { Complexity }\end{array}$ \\
\hline Green Nudge & 2011 & Littering & $\begin{array}{l}\text { Public- } \\
\text { Urban }\end{array}$ & I & Footprint leading to the trash can. & $\begin{array}{l}\text { Removing the effort to find the trash } \\
\text { can. }\end{array}$ & Low \\
\hline $\begin{array}{l}\text { Bottle Bank } \\
\text { Arcade }\end{array}$ & 2012 & Recycling & $\begin{array}{l}\text { Public- } \\
\text { Urban }\end{array}$ & Involvement & The bin resembles an arcade game. & $\begin{array}{l}\text { Stimulating curiosity and } \\
\text { competitiveness }\end{array}$ & High \\
\hline Emoticon bin & 2013 & Recycling & $\begin{array}{l}\text { Public } \\
\text { indoor }\end{array}$ & I & $\begin{array}{l}\text { The bin reacts to the user action smiling } \\
\text { and playing game sounds. }\end{array}$ & $\begin{array}{c}\text { Creating empathy and rewarding the } \\
\text { user. }\end{array}$ & Medium \\
\hline Droppit & N.D. & $\begin{array}{l}\text { Cigarette } \\
\text { littering }\end{array}$ & $\begin{array}{l}\text { Public- } \\
\text { Urban }\end{array}$ & I & The bin consists of a street grate. & $\begin{array}{l}\text { Leveraging the habitual behavior of } \\
\text { throwing cigarettes on the ground. }\end{array}$ & Low \\
\hline FUMO & 2014 & $\begin{array}{l}\text { Cigarette } \\
\text { littering }\end{array}$ & $\begin{array}{c}\text { Public } \\
\text { outdoor, } \\
\text { urban }\end{array}$ & Involvement & $\begin{array}{l}\text { The bin displays flashing lights and } \\
\text { plays music when a cigarette butt is } \\
\text { collected. }\end{array}$ & $\begin{array}{l}\text { Rewarding and amusing the user, } \\
\text { stimulating curiosity. }\end{array}$ & High \\
\hline BinCam & 2015 & $\begin{array}{l}\text { Recycling } \\
\text { Food } \\
\text { waste }\end{array}$ & Private & $\begin{array}{c}\text { Pressure/ } \\
\text { Involvement }\end{array}$ & $\begin{array}{l}\text { The bin is connected to social networks } \\
\text { sharing users' behavior. }\end{array}$ & $\begin{array}{l}\text { Leveraging the need of social } \\
\text { acceptance and awareness of others' } \\
\text { behavior. }\end{array}$ & $\begin{array}{c}\text { Low (in } \\
\text { context) }+ \\
\text { High (App) }\end{array}$ \\
\hline $\begin{array}{c}\text { Transparent trash } \\
\text { bin }\end{array}$ & 2015 & Recycling & Public & Pressure & $\begin{array}{c}\text { The transparent bin allows to see the } \\
\text { content. }\end{array}$ & $\begin{array}{l}\text { making evident the user behavior and } \\
\text { easier to understand disposal. }\end{array}$ & Low \\
\hline Trash Game & 2015 & Recycling & $\begin{array}{l}\text { Public } \\
\text { indoor }\end{array}$ & $\begin{array}{c}\text { Pressure/ } \\
\text { Involvement }\end{array}$ & $\begin{array}{l}\text { The bin is connected to an application } \\
\text { sharing the litter picture and asking } \\
\text { players if the disposal was correct. }\end{array}$ & $\begin{array}{l}\text { Educating crowds about correct } \\
\text { disposal. }\end{array}$ & $\begin{array}{c}\text { Low (in } \\
\text { context) }+ \\
\text { High (App) }\end{array}$ \\
\hline $\begin{array}{l}\text { World's deepest } \\
\text { bin }\end{array}$ & 2015 & Littering & $\begin{array}{l}\text { Public- } \\
\text { Urban }\end{array}$ & Involvement & $\begin{array}{c}\text { The bin plays a sound when a litter is } \\
\text { thrown in it. }\end{array}$ & $\begin{array}{l}\text { Rewarding and amusing the user, } \\
\text { stimulating curiosity. }\end{array}$ & Medium \\
\hline $\begin{array}{l}\text { Anthropomorphic } \\
\text { bin }\end{array}$ & 2016 & Recycling & N.D. & I & $\begin{array}{l}\text { The bin reacts to the user actions } \\
\text { bowing, moving and shaking. }\end{array}$ & Creating empathy towards the bin. & Medium \\
\hline Ballot Bin & 2016 & $\begin{array}{l}\text { Cigarette } \\
\text { littering }\end{array}$ & $\begin{array}{l}\text { Public- } \\
\text { Urban }\end{array}$ & Involvement & $\begin{array}{l}\text { Proposing to users a voting system } \\
\text { concerning users' opinions. }\end{array}$ & $\begin{array}{c}\text { Leveraging self-identity, membership } \\
\text { and values. }\end{array}$ & Low \\
\hline
\end{tabular}

The Green Nudge (Hansen, 2012), the Ballot Bin (https://ballotbin.co.uk/) and DropPit (https://www.thedroppit.eu/) require a simple interaction. Examples of medium level interaction are the World's deepest bin (Kim, 2015) and the Emoticon Bin (Berengueres et al., 2013), which provide feedback raising people's curiosity or emotion, but the feedback is constant and the gestures required by users are usual. Specifically, users can accomplish the usual gestures of throwing litters into the bin, they are not required to execute the task in a different way. Conversely, in the case of the Bottle Bank Arcade (Kim, 2015) and Tetra Bin (http://www.tetrabin.com/), people are encouraged to play and compete with each other. In these cases the action of throwing litters is different from a usual situation (e.g. users have to aim at something, or do it in a specific moment to win the game. However, these strategies can easily be used only in the outdoor urban context. The Tetra Bin (http://www.tetrabin.com/) can better work in certain areas of the city and in the evening, when people are more likely to be around for leisure. FUMO (https://www.fastcompany.com/3030479/meetfumo-the-silliest-possible-way-to-fix-cigarette-littering) is designed for public spaces and its variety and playfulness of feedback leads people to spend more time using the bin, also collecting others' cigarette butts to do it. Using this kind of interactive and captivating solutions is indeed popular in public spaces, while strategies for behavior change in the private context leverage other aspects, such as personalization of feedbacks and suggestions adapting to the users' needs and preferences, as proposed by Monici et al. (2017). However, playful, eye catching and noisy designs may not be the ideal solution for some public outdoor locations requiring quiet, as in proximity of hospitals, since they might be considered disrespectful. 
A possible solution would be to keep the interaction simple in the context where the bin or ashtray is used, transferring involvement strategies in a virtual space (Thieme et al., 2012; Lessel et al., 2015). In these cases, users interacting with the bin are not the only ones involved. In fact, a community of users is also participating through applications and social media.

\section{DESIGN AND DEVELOPMENT OF AIM}

We named the interactive device as AIM to recall an analogy with our design concept. In fact, it stimulates users to throw their cigarette butts by aiming at the center of the ashtray. Thus, this name is used to refer to the current project and the interactive prototype in the following.

\subsection{Concept design}

Considering the previous analysis, we based AIM on social involvement strategies and high interaction level. This approach is appropriate to this case study since the behavior is related to the smoking activity in the campus, therefore it is social (it mostly happens in small groups) and takes place in the outdoor environment. As DropPit (https://www.thedroppit.eu/), AIM uses the strategy of turning a wrong behavior (throwing cigarette butts on the ground) into a correct one but adopting a gamification approach, similarly to the Bottle Bank Arcade (Kim, 2015). Specifically, the initial design idea of AIM is inspired by the design principle of the darts game: the smoker has to aim at the center when disposing cigarette butts for obtaining rewards. AIM includes three areas with corresponding holes in which the cigarette butt can be thrown. According to the hole in which the cigarette butt falls, a different audio feedback is provided: if the user hits the center hole, the receptacle generates a winning sound. The selected sounds in the current project are from the vintage game SUPER MARIO BROS (http://www.nintendo.com/) because of its familiarity and acceptance among a large scale of generations.

A potential issue regards the fact that even though we aim at stimulating a correct and pro-environmental behavior, it is still bonded to the harmful smoking behavior, which should not be encouraged. For this reason, we decided to limit the playful interaction only to the specific action of disposal.

Figure 1 illustrates the overall user interaction procedure with the designed trash bin. Smokers should first put out the cigarette butts in a small container mounted on a pole with a signal placed aside which reports the sentence "PUT OUT and AIM!". Then, they have to throw the cigarette butts into the receptacle, aiming at the red center of the container. When the cigarette butt hits the bottom part of the ashtray, it stimulates a rewarding audio according to the targeted circle.

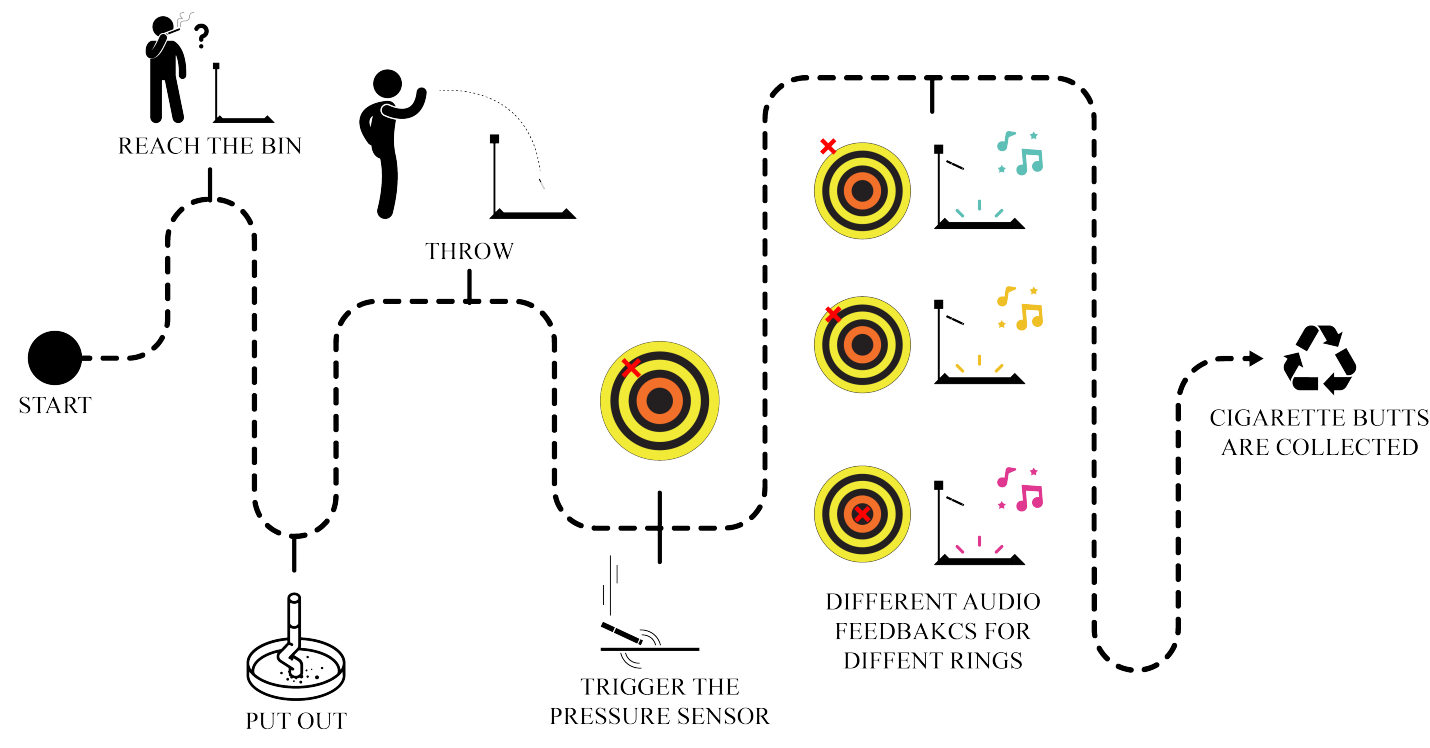

Figure 1. User interaction process with the AIM 


\subsection{Prototype design}

The prototype includes a pressure sensitive conductive material called Velostat (https://www.adafruit.com/ product/1361) to detect the cigarette butts, an Arduino Uno board (https://www.arduino.cc/), a DF Player module (https://www.robotics.org.za/MP3-PLAY), an SD card, a speaker and a 9 Volt battery. The Velostat was placed between two conductive layers of aluminum foil and we adopted the Arduino board to test and control the pressure sensitivity, carrying out multiple sensor calibrations. In the final prototype, the Arduino board is used to read the data from the Velostat and send audio feedbacks through the speaker. The DF Player module is used to manage the communication between the Arduino board and the speaker. The SD card containing the three audio feedbacks is installed into the DF Player. In a first phase, we implemented a rapid prototype by using cardboard as the cigarette filter, to verify that the cigarettes could enter the container and then hit the sensor. In the second phase, we further adjusted the prototype. We divided the filter into three areas, designed with the three inner outward-dipping surfaces, to decrease the number of bounced-off cigarettes. A ring-shaped coverage that surrounds the whole structure was added to prevent cigarettes from deflecting outside. Three different areas in the bottom part are also indicated by three layers of different colored materials which can be distinguished from the upper visible part of the receptacle. Finally, we mounted a small container on a pole attached to the ashtray, with a signal reminding to put off the cigarette before using AIM, as described in Section 4.1. Details of the main components of the prototype can be found in Figure 2.

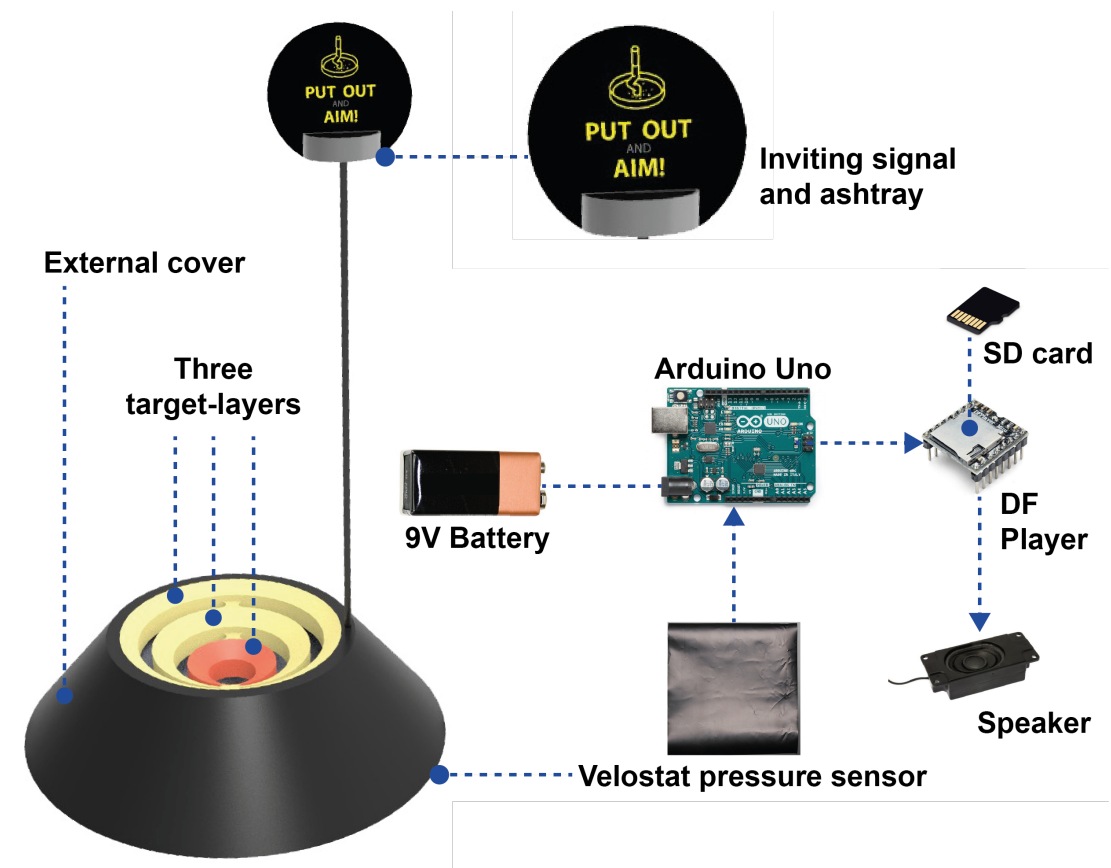

Figure 2. Main components and overall look of the prototype AIM

\section{FIELD STUDY AND USER TEST}

The study followed a 2 (two smoking areas on campus) x 2 (no intervention vs. with intervention) between-subjects test design. The details are described as follow.

\subsection{Objective and hypothesis}

The main objectives of the tests were (1) to observe the actual situation of cigarette butts littering among student smokers in the campus for a specified period of time and to understand the possible reasons behind; (2) to test the efficiency of our interactive ashtray, in order to reduce the littering behaviors. In addition, we hypothesized that (H1) the designed interventions will affect littering behavior among student smokers compared to original conditions; (H2) the effectiveness of behavior change will not only be influenced by the elements embedded in the prototype itself, but also by determinants like intrinsic characters of participants, as well as the extrinsic or contextual factors such as the level of environmental cleanliness. 


\subsection{Sample and procedure}

156 students-smokers were observed in two campus locations in two same days of two weeks. The selection of observing areas was based on the most significant smoking locations of the university. We use $A$ to name the test location in design campus and $B$ to name industrial engineering campus, as shown in Figure 3. The prototype kept the same distance from the trash bins. The time duration for each observation session was one and a half hour: for location $A$, two sessions were conducted from $2: 10 \mathrm{pm}$ to $3: 40 \mathrm{pm}$; for location $B$, from $4: 05$ to $5: 35$.
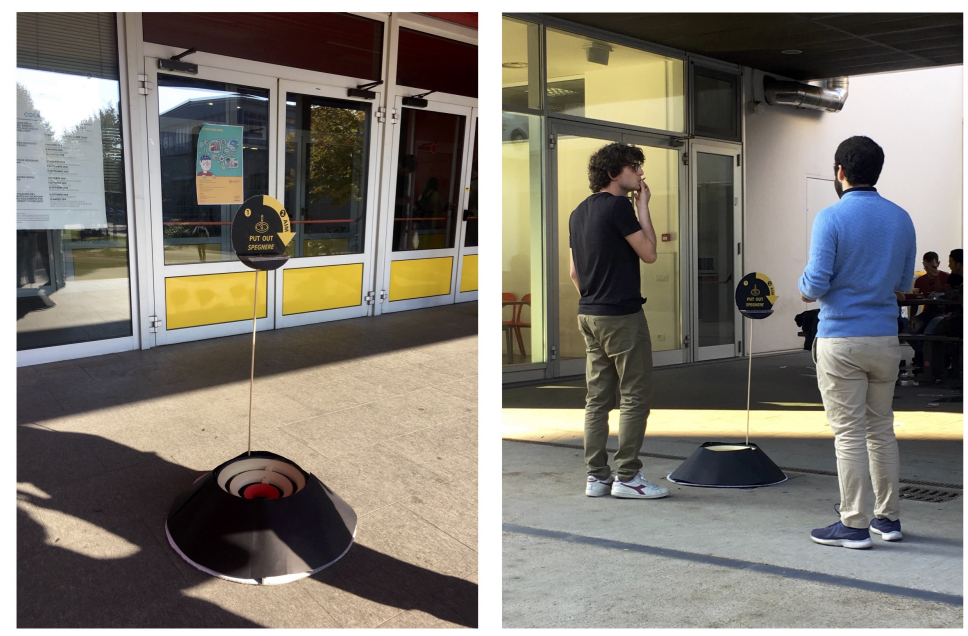

Figure 3. User study in two campus locations (Left: location A; Right: location B).

During the study, two observers were hidden from the observation area while recording, in order to prevent exerting any influence, as well as avoiding idiosyncrasies and personal bias. In the meanwhile, another experimenter was writing explanatory notes and conducting interviews. Randomly selected student-smokers in the observation area were kindly inquired for interviews. The same measurement was repeated in the four study sessions (two locations in two conditions: with and without the prototype) for comparisons.

\subsection{Measurement and material}

The measurement includes two main parts: (1) field observations; and (2) a self-rated questionnaire concerning user experience together with a survey interview. Behavior coding sheet and other related materials are provided for two recorders in order to carry out the study in a standardized way. The data collected in the field observation were environmental cleanness (rated in a scale by two observers from 0 -not clean at all to 10-completely clean) and the number of smokers, as well as their gender distribution. In each session (with-without the prototype), the interviewer recorded 8 interviewees' demographic and personal information like age, gender, and major, as well as their habits regarding smoking. The 8 interviewees in the section with the prototype filled a questionnaire containing 12 pairs of subjective ratings, which was selected from the original User Experience Questionnaire (https://www.ueq-online.org/). We chose the most relevant pairs for our case study (e.g. AIM was designed to be motivating and exciting rather than efficient or fast).

\subsection{Test result}

In Figure 4, the percentage of cigarette butts littering behavior in two campus locations are reported in three aspects: (1) total littering among all the observed student smokers in that session; (2) male smokers' littering among the observed male students, and (3) female smokers' littering among the observed female students.

As shown by the data, the prototype intervention effectively reduced the amount of wrong behavior in both the locations. In fact, the number of littering behavior falls greatly in location A, especially among the male sample ( $15 \%$ over female sample). In location $B$, the decline rate of male $(15 \%)$ and female $(16 \%)$ is very much close. It may due to the reason that the percentage of male smokers compared to female smokers in location A is 1.5 times, while in location $\mathrm{B}$, the number is doubled. 


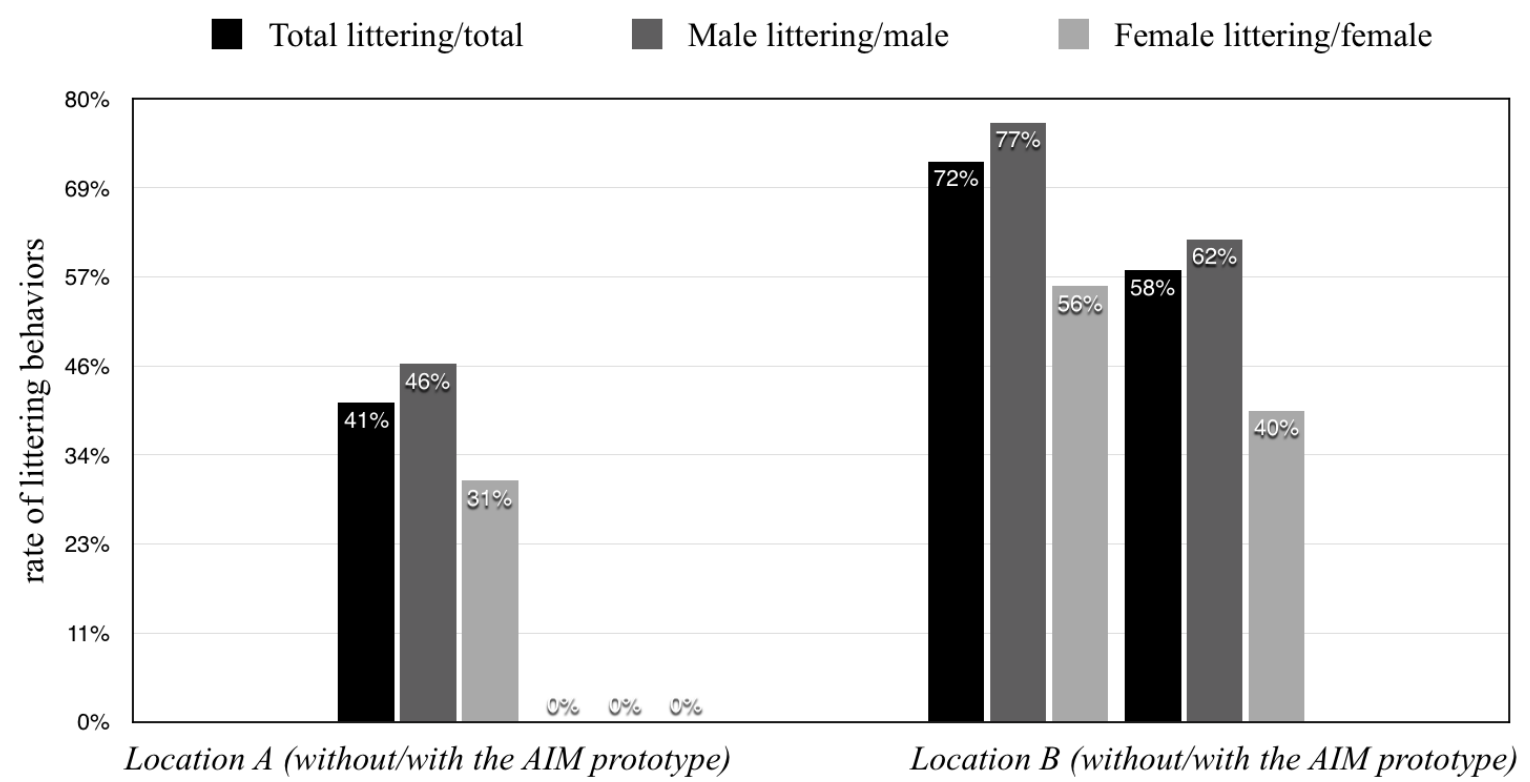

Figure 4. The rate of littering behavior among students-smokers in Locations $A$ (left) and B (right) in the conditions without/with the AIM prototype.

Except for the designed intervention and the participants' involvement, environmental cleanness can also be identified as an important influencing factor in explaining the different littering behavior. The perceived cleanness level of the surrounding environment in location A without the prototype $(M=7.45$; $\mathrm{SD}=0.65)$ together with the prototype $(\mathrm{M}=7.95 ; \mathrm{SD}=1.01)$ are higher than the number in location $\mathrm{B}$ without the prototype $(M=5.18 ; S D=1.18)$ and with the prototype $(M=5.90 ; S D=0.80)$. This result is in line with previous works in the literature concerning human behavior in public space (Schultz et al., 2013; Cialdini et al., 1990).

We performed interview sessions with 16 participants equally distributed in the two locations and conditions (with-without AIM) with an average age of $21.75(\mathrm{SD}=2.27)$. According to the collected data, $87.5 \%$ of smokers stated they usually smoke with friends rather than alone, therefore, most of them are having conversations in the meanwhile. This result reinforces our design idea that in similar cases, a gamified ashtray should stand under an interpersonal context and the interaction should be able to involve multiple users. Considering the reasons of throwing cigarette butts on the ground, the frequently reported answers are related with availability and proximity of the ashtray, in compliance with previous works in literature (Schultz et al., 2013; Bator et al., 2011; Finnie, 1973).

In addition, 8 participants, equally distributed in the two locations, were invited to report their user experience rating it by 12 identified pairs of adjectives. In Figure 5, the blue circle represents the result in location $\mathrm{A}$ and the yellow triangle is for location $\mathrm{B}$, while the limits are indicating the minimum and maximum values in each adjective pair. Five adjective pairs (obstructive-supportive, demotivating-motivating, confusing-clear, unfriendly-friendly, conservative-innovative), the average perception of participants in the two locations is similar. Only for the description pairs boring-exciting and complicated-easy, subjects evaluations are slightly different.

Moreover, all the interviewed students who experienced the prototype stated they were willing to use the new type of interactive ashtray since it is interesting and engaging, especially when smoking together with friends. Lastly, participants provided some suggestions for improvements and implementation of the prototype, such as increasing the scale and size of the product to enhance visibility and adding visual feedback.

\section{CONCLUSION}

Design for behavior change leverages product and service design as an effective medium to enhance pro-environmental and pro-social behaviors. In particular, interactive products can give users inputs and feedbacks, stimulating a behavior in advance and/or providing a response to users' actions. Specifically, 


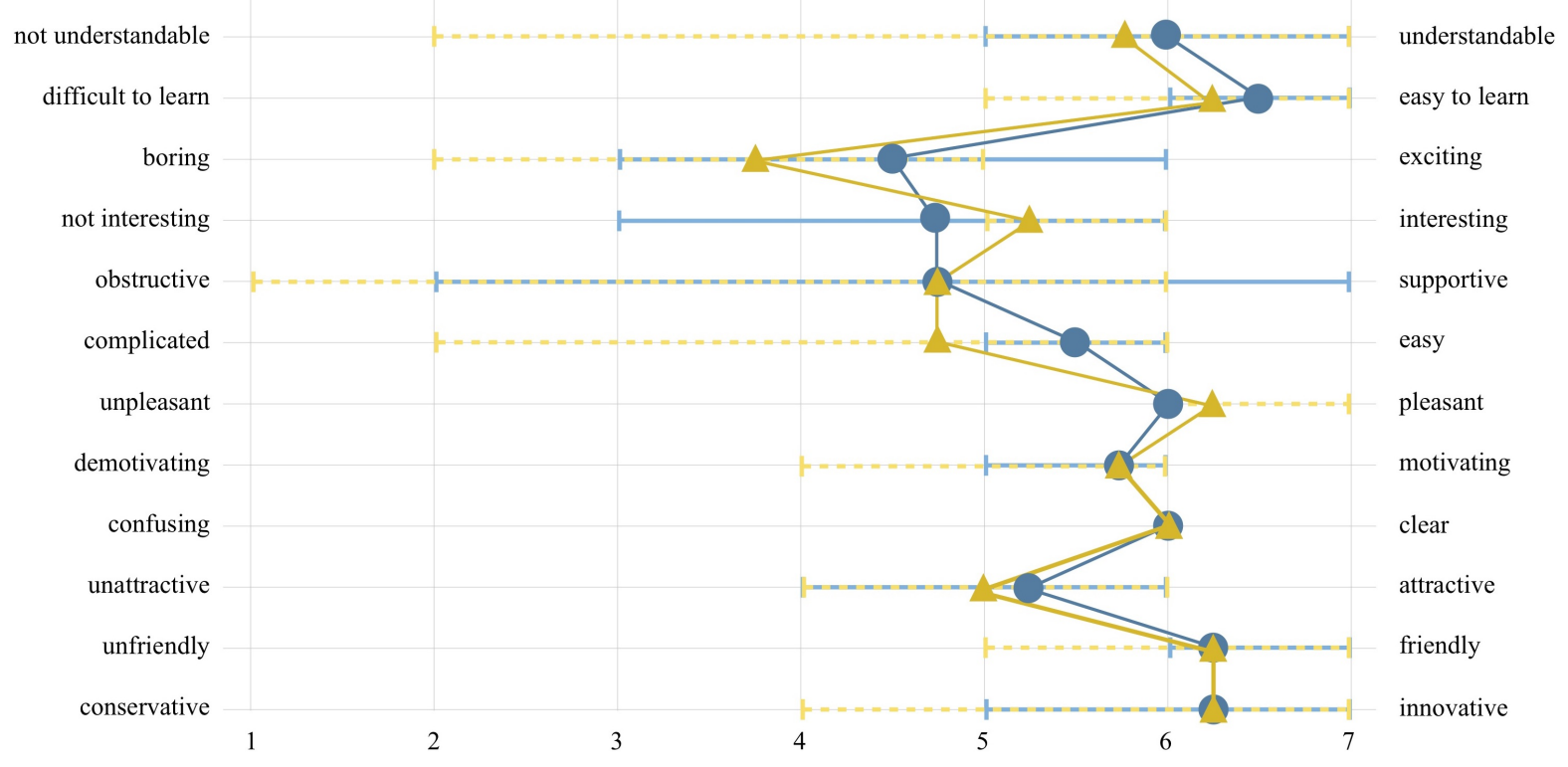

Figure 5. User experience questionnaire result in mean, minimum and maximum values.

game-based elements embedded into the design of products can be used to engage users in a persuasive way.

This work proposes an analysis of previous case studies as a guide to design an interactive ashtray for the university campus environments, to contrast the cigarette butts littering behavior. We used involvement as a social factor and a high level of interaction, as described in Section 3. Different design principles are combined in the design: attracting smokers through the ashtray visual appearance, leveraging their habitual behavior of throwing cigarettes on the ground, rewarding and amusing the participation through gamified elements such as target-setting and audio feedback and addressing the social character of the smoking activity by stimulating gathering and competition. This work is a example of how different identified social factors, interaction levels and design principles can be combined, addressing certain of target users and context: these guidelines can be used to develop more case studies and gamification trends. Considering our context, AIM was suitable for the outdoor campus environment: as it was positioned close to halls or entryways rather than classrooms, it did not disturb any teaching or studying activity. As demonstrated by the results, the behavior of disposers was effectively influenced by their experience with the product even though other factors, as the environmental conditions, meaning the cleanliness of the environment, seemed to affect their behavior as well. However, the validity of this prototype on a long-term scale should be further tested since gamification alone is not proved to maintain an everlasting effect. Therefore, this strategy might be integrated with others, to continue motivating a correct disposal behavior. An example would be the one of keeping the users involved after the smoking and disposal,including the use of applications, social media, and multi-player games, as proposed by other studies (Thieme et al., 2012; Lessel et al., 2015). Other directions for future development include the adaptation, implementation and test of the current prototype for a broader context and different target groups.

\section{REFERENCES}

Bator, R. J., Bryan, A. D. and Schultz, P. W. (2011), "Who gives a hoot?: Intercept surveys of litterers and disposers", Environment and Behavior, Vol. 43 No. 3, pp. 295-315. http://doi.org/10.1177/0013916509356884

Berengueres, J., Alsuwairi, F., Zaki, N. and Ng, T. (2013), "Gamification of a recycle bin with emoticons", in Proceedings of the 8th ACM/IEEE international conference on Human-robot interaction, Tokyo, Japan, 23rd-6th March 2013, IEEE Press, pp. 83-84. http://doi.org/10.1109/HRI.2013.6483512

Cialdini, R. B., Reno, R. R. and Kallgren, C. A. (1990), "A focus theory of normative conduct: recycling the concept of norms to reduce littering in public places.", Journal of personality and social psychology, Vol. 58 No. 6, pp. 1015-1026. http://doi.org/10.1037/0022-3514.58.6.1015 
De Kort, Y. A., McCalley, L. T. and Midden, C. J. (2008), "Persuasive trash cans: Activation of littering norms by design", Environment and Behavior, Vol. 40 No. 6, pp. 870-891. http://doi.org/10.1177/0013916507311035

Deterding, Sebastian and Sicart, Miguel and Nacke, Lennart and O'Hara, Kenton and Dixon, Dan, "Gamification. using game-design elements in non-gaming contexts", in Proceedings of CHI'11 extended abstracts on human factors in computing systems, Vancouver, Canada, 7th-12th ACM May 2011, pp. 2425-2428. http://doi.org/10.1145/1979742.1979575

Finnie, W. C. (1973), "Field experiments in litter control”, Environment and behavior, Vol. 5 No. 2, pp. $123-144$. http://doi.org/10.1177/001391657300500201

Fogg, B. J. (2009), “A behavior model for persuasive design", in Proceedings of the 4th international Conference on Persuasive Technology, Claremont, California, USA, 26th-29th April 2009, ACM, No. 40. http://doi.org/10.1145/1541948.1541999

Geller, E. S., Brasted, W. S. and Mann, M. F. (1979), "Waste receptacle designs as interventions for litter control”, Journal of Environmental Systems, Vol. 9 No. 2, http://doi.org/10.2190/5P46-8H2N-41JR-C2EJ

Hansen, P. (2012), 'inudgeyou green nudge: Nudging litter into the bin-inudgeyou, http://inudgeyou. com/green-nudge-nudging-litter-into-the-bin. (Last checked 04 February 2019).

https://www.adafruit.com/product/1361 (Last checked 26 November 2018).

https://www.arduino.cc/ (Last checked 26 November 2018).

https://ballotbin.co.uk/(Last checked 6 September 2018).

https://www.fastcompany.com/3030479/meet-fumo-the-silliest-possible-way-to-fix-cigarette-littering (Last checked 26 November 2018).

http://www.nintendo.com/ (Last checked 26 November 2018.).

https://www.robotics.org.za/MP3-PLAY (Last checked 26 November 2018.).

http://www.tetrabin.com/ (Last checked 06 September 2018.).

https://www.thedroppit.eu/ (Last checked 06 September 2018.).

https://www.ueq-online.org/ (Last checked 26 November 2018.).

Jager, W. (2003), 'Breaking bad habits: a dynamical perspective on habit formation and change', Human Decision-Making and Environmental Perception-Understanding and Assisting Human Decision-Making in Real Life Settings. Libor Amicorum for Charles Vlek, Groningen: University of Groningen.

Kim, B. (2015), "Designing Gamification in the Right Way", in Kim, B. Library Technology Reports, ALA TechSource, Vol. 51 No. 2, pp. 10-18.

Lessel, P., Altmeyer, M. and Krüger, A. (2015), “Analysis of recycling capabilities of individuals and crowds to encourage and educate people to separate their garbage playfully", in Proceedings of the 33rd Annual ACM Conference on Human Factors in Computing Systems, Seoul, Republic of Korea, 18th-23rd April 2015, ACM, pp. 1095-1104. http://doi.org/10.1145/2702123.2702309

Matsumura, N., Fruchter, R. and Leifer, L. (2015), "Shikakeology: designing triggers for behavior change", $A I$ \& SOCIETY, Vol. 30 No. 4, pp 419-429. http://doi.org/10.1007/s00146-014-0556-5

Monici, D., Graziosi, S., Ferrise, F., Bordegoni, M. et al. (2017), "Design of a smart alarm clock to foster sustainable urban mobility", in DS 87-8 Proceedings of the 21st International Conference on Engineering Design (ICED 17) Vol 8: Human Behaviour in Design, Vancouver, Canada, 21st-25th August 2017, pp. 359-368. http://doi.org/10.1016/j.tranpol.2010.08.006.

Montazeri, S., Panos, P., Rich, G. et al. (2015), "Design for behavior change: An elaboration-based approach to persuasion in product design", in DS 80-11 Proceedings of the 20th International Conference on Engineering Design (ICED 15), Milan, Italy, 27th-30th July 2015, pp. 001-010.

Rath, J. M., Rubenstein, R. A., Curry, L. E., Shank, S. E. and Cartwright, J. C. (2012), "Cigarette litter: smokers' attitudes and behaviors", International journal of environmental research and public health, Vol. 9 No. 6 , pp. 2189-2203. http://doi.org/10.3390/ijerph9062189

Schultz, P. W., Bator, R. J., Large, L. B., Bruni, C. M. and Tabanico, J. J. (2013), "Littering in context: personal and environmental predictors of littering behavior", Environment and Behavior, Vol. 45 No. 1, pp. 35-59. http://doi.org/10.1063/1.4982841

Tan, H., Sun, L. and Šabanović, S. (2016), "Feeling green: Empathy affects perceptions of usefulness and intention to use a robotic recycling bin", in Robot and Human Interactive Communication (RO-MAN), New York, USA, 26th-31st August 2016, IEEE, pp. 1051-1056. http://doi.org/10.1109/ROMAN.2016.7745238

Thieme, A., Comber, R., Miebach, J., Weeden, J., Kraemer, N., Lawson, S. and Olivier, P. (2012), “We've bin watching you: designing for reflection and social persuasion to promote sustainable lifestyles", in Proceedings of the SIGCHI Conference on Human Factors in Computing Systems, Austin, Texas, USA, 5th-10th May 2012, ACM, pp. 2337-2346. http://doi.org/10.1145/2207676.2208394

Wever, R., Van Onselen, L., Silvester, S. and Boks, C. (2010), "Influence of packaging design on littering and waste behaviour", Packaging Technology and Science, Vol. 23 No. 5, pp. 239-252.

http://doi.org/10.1002/pts.892. 